\title{
LINESHAPE MEASUREMENTS OF ROTATIONAL LINE IN MILLIMETER-WAVE REGION BY SECOND HARMONIC DETECTION
}

\author{
J. Stanek, S. Gierszal and J. Galica \\ Institute of Molecular Physics, Polish Academy of Sciences \\ Smoluchowskiego 17, 60-179 Poznań, Poland
}

(Received November 17, 1992; revised version August 26, 1993)

\begin{abstract}
Lineshape measurements of the $J=6 \leftarrow 5$ OCS rotational transition were performed using a source-modulation microwave spectrometer. This is a very useful experimental technique but it introduces distortions to the line profile, therefore several authors have theoretically analyzed this problem with the aim of extracting the undistorted parameters of the spectra. The objective of this paper is to verify one of these thearies which gives the relationship between harmonic signals and lineshape over wide range of modulation depth. Detailed comparison between the calculated and experimental harmonic signals shows very good agreement, and thus enables us to determine the unmodified line width and finally the pressure broadening factor of the rotational transition $J=6 \leftarrow 5$ of OCS molecule to be equal to $6.59 \pm 0.25 \mathrm{MHz} / \mathrm{Tr}$.
\end{abstract}

PACS numbers: $33.20 . \mathrm{Bx}, 33.70 . \mathrm{Jg}$

\section{Introduction}

One of the methods of measurement of the absorption lines widely accepted for its great effectiveness in measuring weak signals is the detection of the second harmonic of the modulation frequency.

In lineshape studies it is essential that, in addition to the corrections due to the Doppler effect, the path length of the absorption, collision broadening and saturation effects, the influence of the frequency modulation is also accounted for [1]. The first attempts to describe the effects of the modulation broadening of the Lorentzian line are due to Karplus who in 1948 published the theoretical analysis of the absorption lineshape [2].

These effects have been more thoroughly analyzed later on by many researchers, among others by Reid and Labrie [3], who made a similar analysis 
including also the Gaussian and Voigt shape functions and performed a broad comparison of the experimental data with their theoretical models.

In the present study a method of the measurement of the linewidth, at several gas pressures, is described in which the effect of the double modulation is accounted for. The correctness of these measurements is checked by the determination of the line broadening coefficient of the $J=6 \leftarrow 5$ line of OCS molecule in gas phase.

\section{Model}

In the gas pressure range used in the microwave measurements of molecules in gaseous phase in the case when the pressure broadening predominates other factors, the absorption line can be adequately described by a Lorentzian function

$$
L(\nu)=\pi^{-1}\left(\frac{\Delta \nu}{(\Delta \nu)^{2}+\left(\nu_{0}-\nu\right)^{2}}\right),
$$

where $\Delta \nu$ is half-width measured at the half height (HWHH) of the line centered at $\nu_{0}$.

In the case of a sinusoidal source modulation at a frequency $\nu$, as used in the experiment, the time dependence is of the form

$$
\nu(t)=\nu+\nu_{\omega} \cos \left(\omega_{\mathrm{m}} t\right)
$$

where $\nu_{\omega}$ is the modulation depth and $\omega_{\mathrm{m}}$ is the modulation frequency.

Under the assumption that the frequency $\nu$ does not change during the modulation period, i.e. in the situation when the frequency sweep is sufficiently slow, we may expand the function $L[\nu(t)]$ into the Fourier series

$$
L[\nu(t)]=\frac{\Delta \nu}{2 \pi} \sum_{n=0}^{\infty} a_{n}\left[\Delta \nu, \nu_{\omega}, \nu\right] \cos (n \omega t),
$$

where

$$
a_{n}=\frac{\omega}{\pi} \int_{-\pi / \omega}^{\pi / \omega} \frac{\cos (n \omega t)}{(\Delta \nu)^{2}+\left[\left(\nu-\nu_{0}\right)+\nu_{\omega} \cos (\omega t)\right]^{2}} \mathrm{~d} t
$$

is directly proportional to the signal detected at the diode. The analytical solution of this integral was given by Wahlquist [4] and for $n=2$, it takes the following form:

$$
a_{2}=\left(\frac{2}{\nu_{\omega}}\right)^{2}\left[1+\frac{u^{1 / 2}(1+2 \gamma-2 u)}{2(u-2)^{1 / 2}(u-\gamma)}\right]
$$

where the meaning of the substitutions and definitions is given below

$$
\begin{aligned}
& \alpha=\left(\nu-\nu_{0}\right) / \nu_{\omega}, \quad-\infty<\alpha<\infty, \\
& \beta=(\Delta \nu) / \nu_{\omega}, \quad 0<\beta<\infty, \\
& \gamma=1+\beta^{2}+\alpha^{2}, \quad u=\gamma+\left[\gamma^{2}-4 \alpha^{2}\right]^{1 / 2}, \quad 2<u<\infty .
\end{aligned}
$$

These equations are used to obtain the theoretical dependence of the linewidth as a function of the modulation depth $\nu_{\omega}$ in the situation when the unperturbed linewidth is known. Typical form of $a_{2}(\nu)$ dependence for various magnitudes of the modulation depth is plotted in Fig. 1. The resonance frequency $\nu_{0}=$ $72976.7794 \mathrm{MHz}$ was adopted from [5] and $\Delta \nu=0.358921 \mathrm{MHz}$ was taken as the value of the linewidth, unaffected by the modulation. 


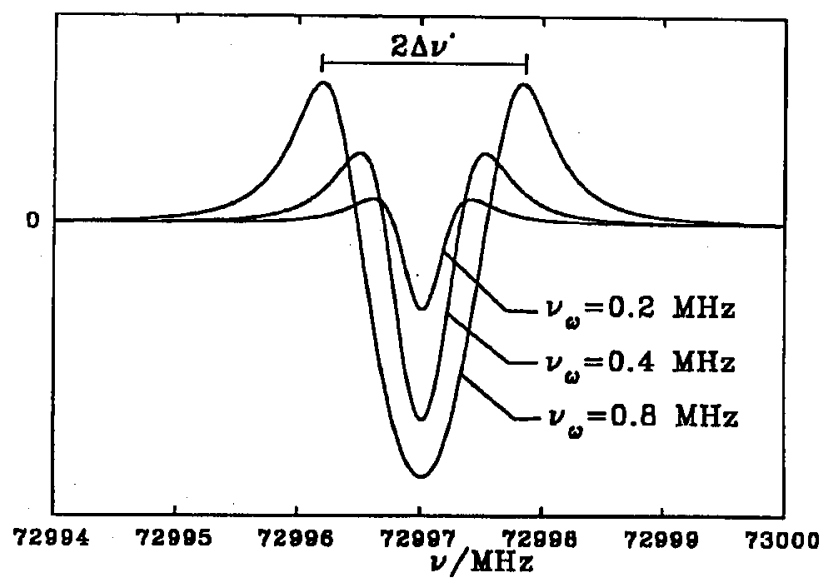

Fig. 1. Three second harmonic lines at different modulation depths $\nu_{\omega}$.

\section{Instrumental}

Figure 2 shows the millimeter band double modulation spectrometer used in the measurements. The generator of the electromagnetic wave is a K766 klystron

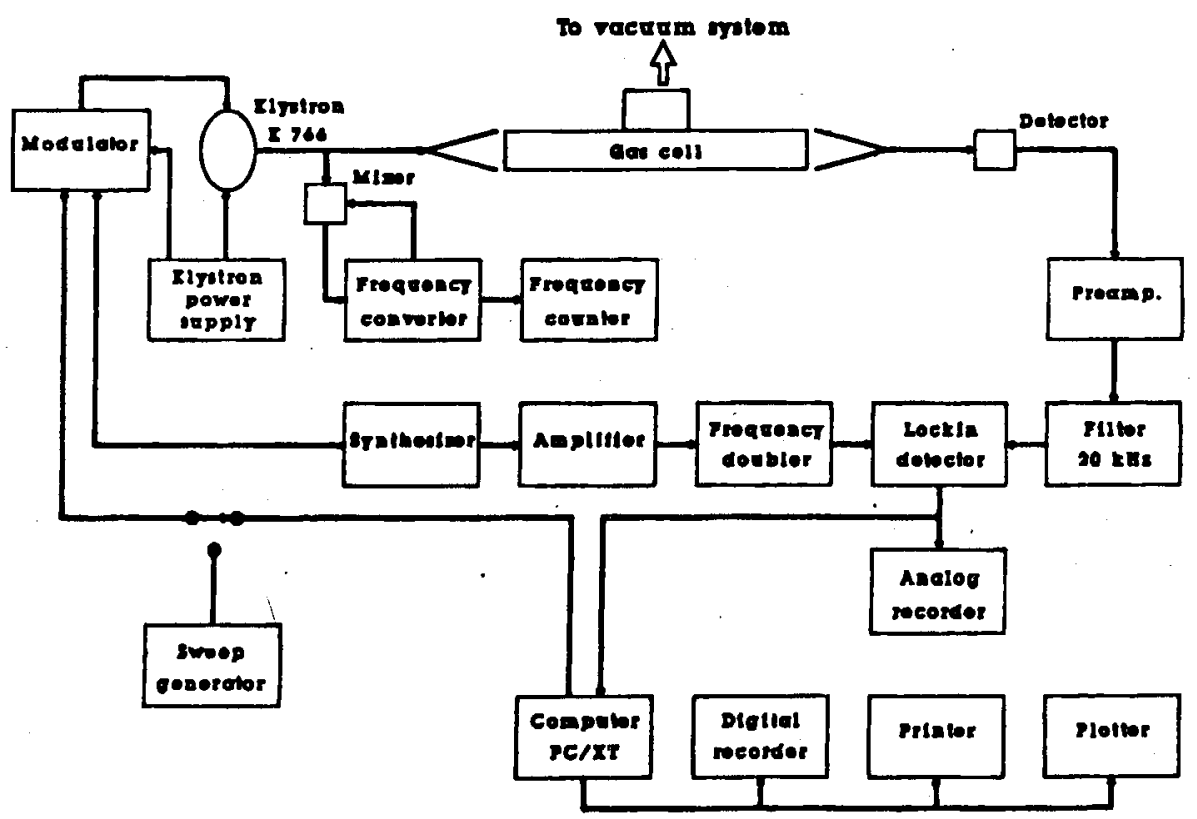

Fig. 2. Block diagram of the millimeter-wave spectrometer. 
integrated with an oil tank and a heat exchanger. The oil filled tank with high voltage bushings for the klystron electrodes is closed with a cover providing for the coarse microwave frequency regulation and with a revolution counter as well as a microwave output. The generated signal is voltage-modulated at $10 \mathrm{kHz}$ frequency and the klystron frequency is slowly swept over $10 \mathrm{MHz}$ range with the linearity $0.001 \%$ at the repetition time of 90 seconds. The electromagnetic wave sent through the transmitter antenna, irradiates the microwave absorption cell made of glass cylinder of length $1.4 \mathrm{~m}$ and of $0.1 \mathrm{~m}$ diameter and closed tightly with teflon windows at both ends. Vacuum installation provides a base pressure of $2.5 \times 10^{-6} \mathrm{Tr}$. The gas under investigation was admitted into the absorption cell at different pressures in the range of 11-33 mTr. During the recording of the spectrum the pressure of the gas was stable within $0.1 \mathrm{mTr}$. The sample pressures were measured by means of a MKS Baratron type $220 \mathrm{C}$ pressure gauge. The absorption signal is received by a millimeter wave point contact detector (MWD) sensitive in the frequency range from 70 to $75 \mathrm{GHz}$. The demodulated signal from MWD passes to a low noise preamplifier, followed by a broadband amplifier producing the signal to be monitored on the oscilloscope. The measurement of the lineshape is performed with a phase-sensitive detector (PSD) and a graphic recorder. The input sensitivity of the detection system is of the order of microvolts and the bandwidth is controlled by the time constant of PSD variable between 1 and 10 seconds. The phase detector drives one of the inputs of a two-channel recorder of $X T$-type, the other channel serves as the input for the frequency calibration marks generated in the spectrometer control system. The spectrometer is completed with a PC/XT computer allowing signal accumulation through AD/DA converter and its numerical analysis as well as the control of the setup of the transmitter.

\section{Linewidth measurement and discussion of the results}

Systematic linewidth measurements $\Delta \nu$ were performed for OCS at natural isotopic abundance by studying the transition $J=6 \leftarrow 5$ in gas phase at several pressure values between 11 and $33 \mathrm{mTr}$, at a temperature of $300 \mathrm{~K}$. The microwave absorption signal recorded by the spectrometer is in the form of second derivative of Lorentzian curve and the linewidth $\Delta \nu^{\prime}$ is measured between the two outer extrema of the curve. To eliminate the reflections due to standing waves the symmetry of the line was used for minimizing the reflection effects. Amongst the parameters characterizing the shape of this curve, its linewidth (which is of our interest) contains the information about intermolecular interactions. The value measured in an experiment $\Delta \nu^{\prime}$, apart from the intrinsic linewidth relating to the nature of the absorption phenomenon, carries also the instrumental effects. The double frequency modulation used in the experiments plays a major role in the broadening due to the instrumental factors. It is of fundamental importance to determine the extent of these factors in measurements of linewidths. With this aim the linewidth $\Delta \nu^{\prime}$ of the rotational transition $J=6 \leftarrow 5$ of OCS molecule was measured as a function of the voltage which drives the reflector of the klystron. The best-fit curve obtained for the above experimental results was extrapolated down to zero modulation voltage yielding the linewidth $\Delta \nu_{1}$ corresponding to the lineshape not 
affected by the modulation. The value obtained from the extrapolation was then used in the calculation of the linewidth versus the modulation depth dependence described by the theoretical model (Eq. (5)).

Figure 3 shows typical results of the calculations for second derivative of the absorption line together with the residuals, that is, the differences between the experimental data and the theoretical curve. There is a good fit between the Wahlquist model [4] and the experimental points of Fig. 3 which justifies referring to the extrapolated linewidth $\Delta \nu_{1}$ as to the linewidth free of the frequency source modulation effects. Scaling factor $a=22.67 \pm 0.07 \mathrm{MHz} / \mathrm{V}$ was used to fit the theoretical results to the experimental data points.

The corrections due to the microwave absorption cell broadening $\Delta \nu_{\mathrm{L}}$ (Fig. 5) [7], the wall collisions effects $\Delta \nu_{\text {wall }}$ of the order of $0.5 \mathrm{kHz}$, saturation phenomena $\Delta \nu_{\text {sat }}=0.1 \mathrm{MHz}$ [9], harmonic distortion in the modulation $1.56 \%$, nonlinearity of the diode $0.4 \%$, nonlinearity of the frequency scale $3.4 \%$, nonlinearity of the amplification $0.06 \%$ and the Doppler broadening $\Delta \nu_{\mathrm{D}}$ (Fig. 6) [6] are obtained from the extrapolated linewidth $\Delta \nu_{1}$. The linewidth $\Delta \nu_{1}$ varies linearly with the gas pressure (Fig. 4). The Doppler-broadening effect $\Delta \nu_{\mathrm{D}}$ (Fig. 6) [6] was obtained in the following way: the widths for the Voigt line shape were computed numerically with the collision HWHH $\Delta \nu_{\text {coll }}$ ranging from 40 to $400 \mathrm{kHz}$ and the Doppler HWHH $\Delta \nu_{D_{0}}=58.35 \mathrm{kHz}$, obtained from the Maxwell-Boltzmann distribution of velocities [1]:

$$
\Delta \nu_{\mathrm{D}_{0}}=\frac{2 \nu_{0}}{c}\left(\frac{2 N k T \ln 2}{M}\right)^{1 / 2}
$$

where $k$ is the Boltzmann constant, $T$ is the absolute temperature, $M$ is the

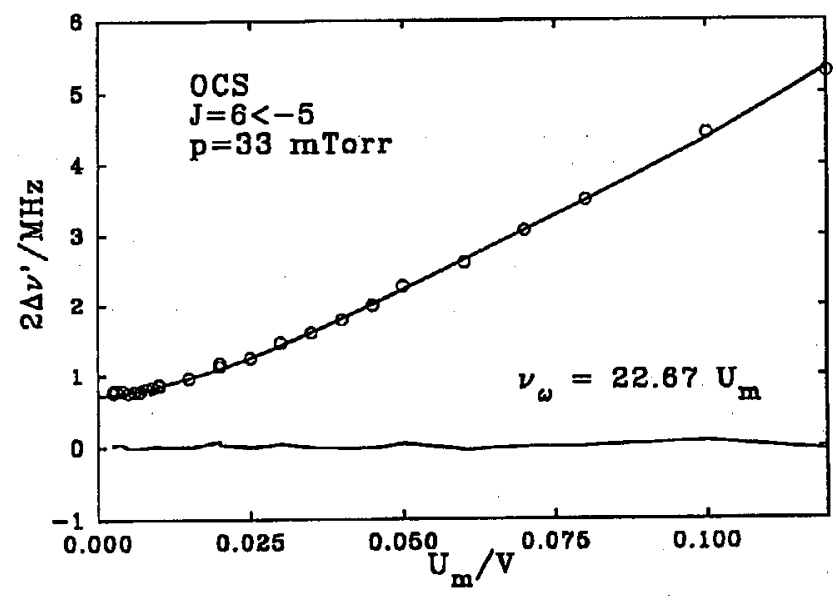

Fig. 3. Wahlquist's theory curve fitted to the experimental data of the linewidth and the residuals versus modulation depth $\nu_{\omega}$. 


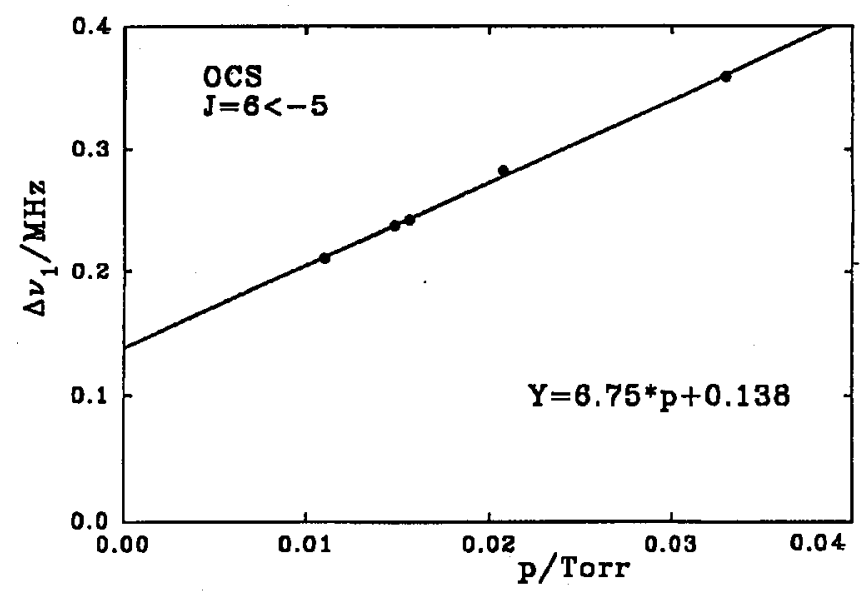

Fig. 4. Linear dependence of the extrapolated linewidth $\Delta \nu_{1}$ versus gas pressure.

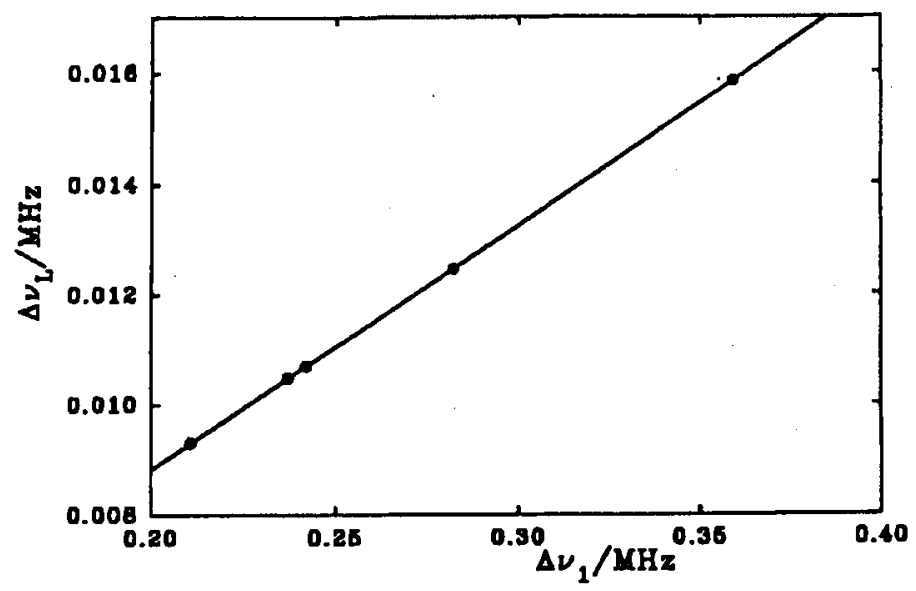

Fig. 5. The change of the linewidth $\Delta \nu_{\mathrm{L}}$ of the rotational transition $J=6 \leftarrow 5$ of OCS molecule as a result of the length $L=1.4 \mathrm{~m}$ of the absorption path.

molecular mass and $N$ is Avogadro's number. The results (i.e., Voigt's width minus collision width) were then numerically fitted to an exponential function by means of a nonlinear least squares routine.

Figure 6 shows the curve of the Doppler corrections versus experimental HWHH $\Delta \nu_{\mathrm{V}}$ corrected previously for all broadening contributions. After taking into account all these corrections one arrives at the value of the linewidth characteristic of the applied gas pressure [10]. The measurements of the linewidth of OCS molecule and the analysis above were performed at selected values of the gas pressure in the pressure range from $11 \mathrm{mTr}$ to $33 \mathrm{~m} T r$. The results of the present study is the value of the broadening coefficient of $\Delta \nu / p$ of the rotational transition 


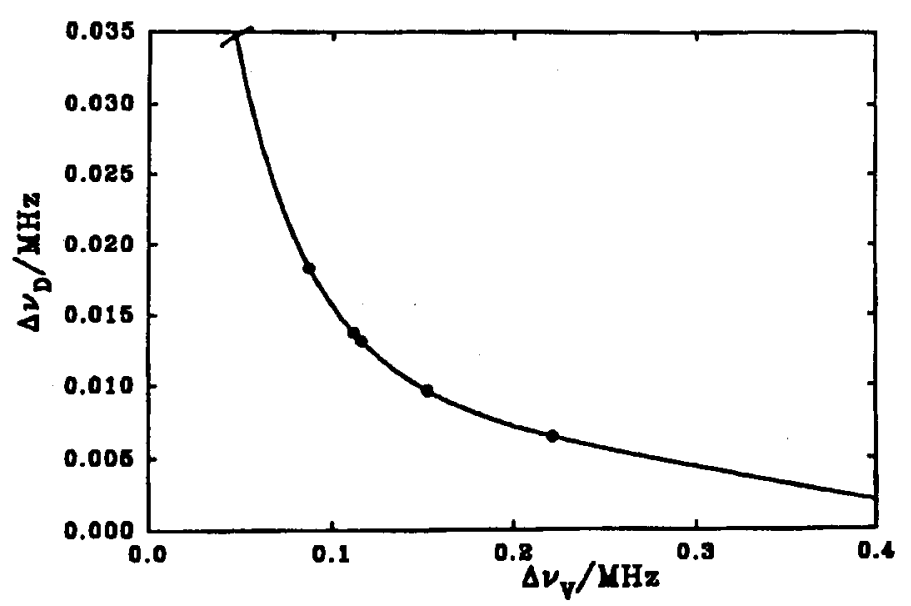

Fig. 6. Doppler-broadening effect on the $J=6 \leftarrow 5$ transition of OCS molecule versus $\Delta \nu_{1}$ linewidth.

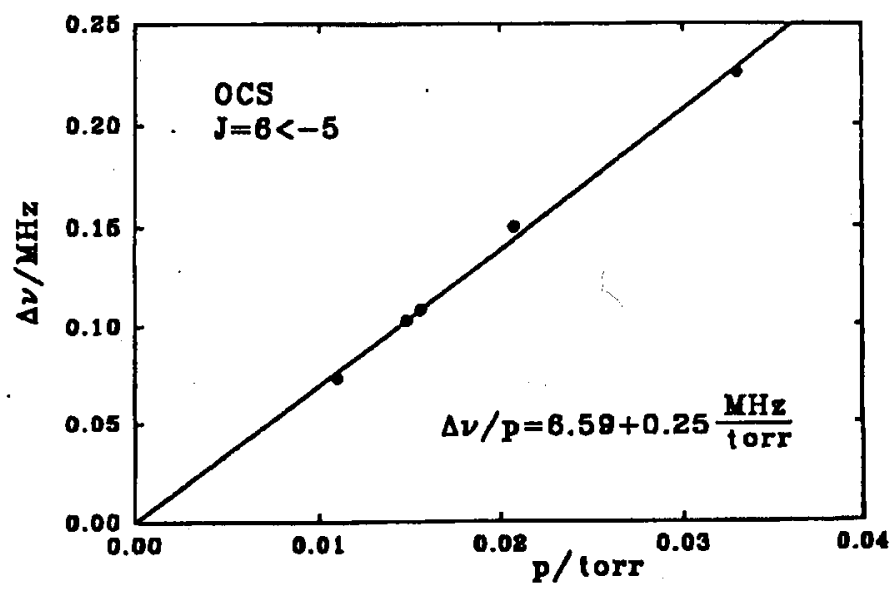

Fig. 7. The linewidth $\Delta \nu$ of the $J=6 \leftarrow 5$ rotational transition of OCS as a function of the gas pressure.

$J=6 \leftarrow 5$ of OCS molecule which is the tangent of the line of the least squares fit (Fig. 7). The value of this coefficient obtained at the correlation factor $r^{2}=0.9958$ is $\Delta \nu / p=6.59 \pm 0.25 \mathrm{MHz} / \mathrm{Tr}$.

\section{Conclusions}

The present value of the broadening coefficient of the $J=6 \leftarrow 5$ transition of OCS molecule is an intermediate of the values quoted from the literature: 
7.9 MHz/Tr [11], 6.52 MHz/Tr [12] and 6.4 MHz/Tr [13]. The mean of the three agrees with our result within the experimental error. Therefore, the result can be accepted at the present state of the metrology in this science. In addition, the results allow to conclude that:

- Wahlquist's theoretical model is applicable in accounting for the dependence of the second derivative of the Lorentzian linewidth on depth of modulation of the microwave source,

- the character of the dependence of the second derivative of the line on depth of the microwave source modulation varies with the applied pressure,

- the extrapolated value of the linewidth $\Delta \nu_{1}$ obtained from the best fit curve in the linewidth vs. modulation voltage characteristic may be regarded as the linewidth free from any frequency modulated distortions,

- extrapolated values of the linewidth $\Delta \nu_{1}$ depend linearly on the gas pressure.

\section{Acknowledgment}

We gratefully acknowledge the financial support of this work provided by the Institute of Molecular Physics of the Polish Academy of Sciences.

\section{References}

[1] J.M. Hollas, High Resolution Spectroscopy, Butterworth \& Co., London 1982.

[2] R. Karplus, Phys. Rev. 74, 1027 (1948).

[3] J. Reid, D. Labrie, Appl. Phys. B 26, 203 (1981).

[4] H. Wahlquist, J. Chem. Phys. 35, 1708 (1961).

[5] A. Dubrulle, J. Demaison, J. Burie, D. Bucher, Z. Naturforsch. 55a, 471 (1980).

[6] R.W. Parsons, J.A. Roberts, J. Mol. Spectrosc. 18, 412 (1965).

[7] E. Rinehart, R.L. Legan, C.C. Lin, Rev. Sci. Instrum. 36, 511 (1965).

[8] S.C.M. Luijendijk, J. Phys. B 8, 2992 (1975).

[9] M.W.P. Strendberg, H.R. Johnson, J.R. Eshbach, Rev. Sci. Instrum. 25, 776 (1954).

[10] S. Gierszal, J. Galica, E. Miś-Kuźmińska, Acta Phys. Pol. A 79, 451 (1991).

[11] R.S. Anderson, Phys. Rev. 97, 1654 (1955).

[12] A. Battaglia, M. Cattani, O. Tarrini, Nuovo Cimento B 61, 193 (1969).

[13] W.F. Kolbe, H. Buscher, B. Leskovar, J. Quant. Spectrosc. Radiat. Transf. 18, 47 (1977). 